\title{
LA-UR-13-23986
}

Approved for public release; distribution is unlimited.

Title:

\section{CMIME Update on Metal-Metal Oxide Atomistic Models}

\author{
Author(s): $\quad$ Valone, Steven M \\ Baskes, Michael I. \\ Allen, Jonathan $\mathrm{R}$. \\ Dunlap, David $\mathrm{H}$. \\ Atlas, Susan R.
}

Intended for:

Scientific Advisor Board review for Center for Materials at Irradiative and Mechanical Extremes

Issued: 2013-05-31

\section{- TosAlamos NATIONAL LABORATORY}

\author{
EST. 1943
}

Disclaimer:

Los Alamos National Laboratory, an affirmative action/equal opportunity employer,is operated by the Los Alamos National

Security, LLC for the National NuclearSecurity Administration of the U.S. Department of Energy under contract DE-AC52-06NA25396. By approving this article, the publisher recognizes that the U.S. Government retains nonexclusive, royalty-free license to publish or reproduce the published form of this contribution, or to allow others to do so, for U.S. Government purposes.

Los Alamos National Laboratory requests that the publisher identify this article as work performed under the auspices of the

U.S. Departmentof Energy. Los Alamos National Laboratory strongly supports academic freedom and a researcher's right to publish; as an institution, however, the Laboratory does not endorse the viewpoint of a publication or guarantee its technical correctness. 


\title{
CMIME Update on Metal-Metal
}

\section{Oxide Atomistic Models}

\author{
Steve Valone
}

LANL

Mike Baskes, UCSD \& LANL

Jon Allen, Prof. Dave Dunlap, Prof. Susan Atlas

UNM Physics

Acknowledgement

Ben Liu

Ghanshyam Pilania

Josh Gibson, UNT 


\section{Capability Gap: \\ Multiple Material Types with One Model}
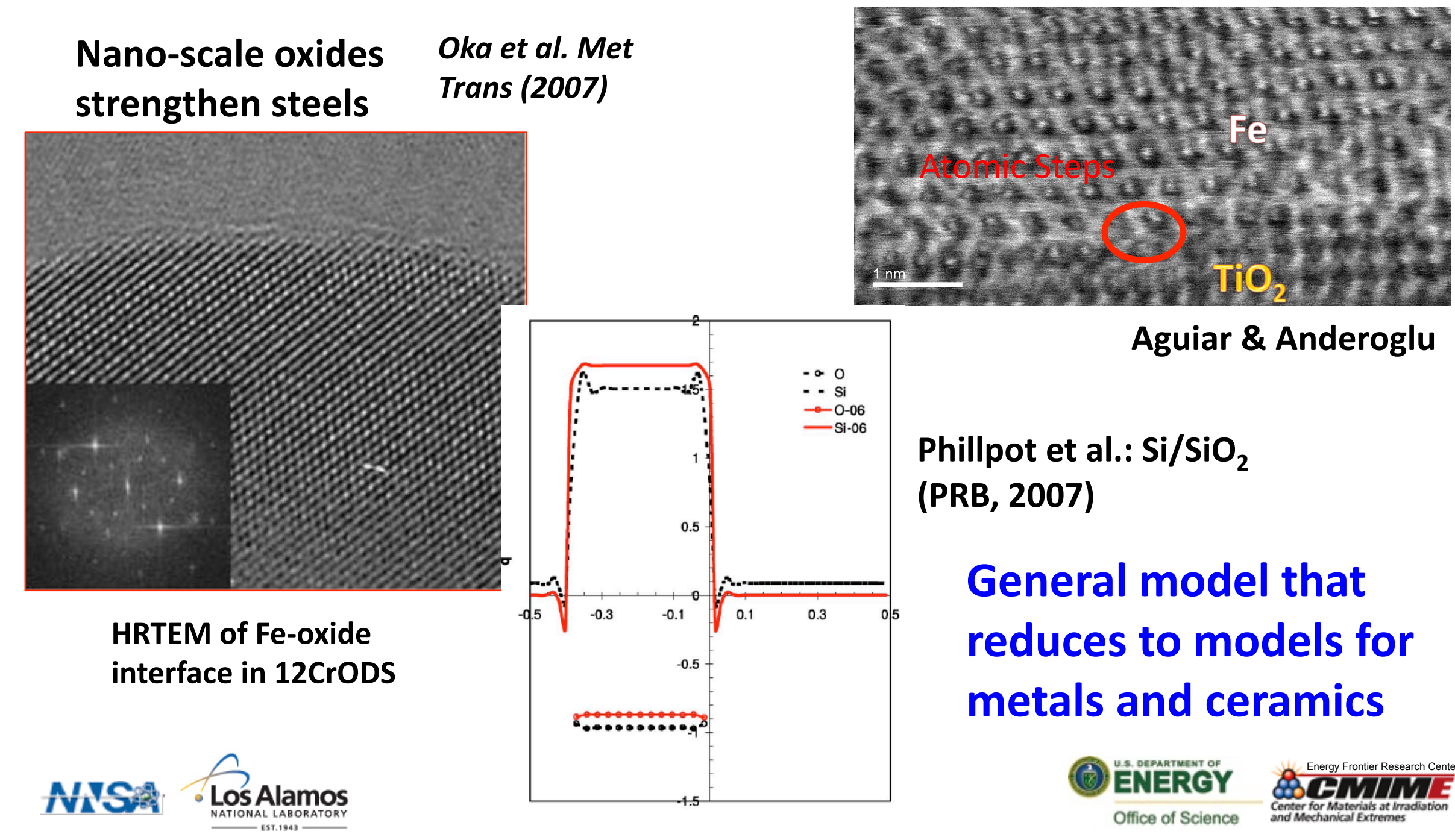

Aguiar \& Anderoglu

Phillpot et al.: $\mathrm{Si} / \mathrm{SiO}_{2}$

(PRB, 2007)

General model that reduces to models for metals and ceramics

(ㄱ) ĖNERGY

Energy Fonitier Research Cente 


\section{Target Applications: Metal/Metal Oxide Composites}

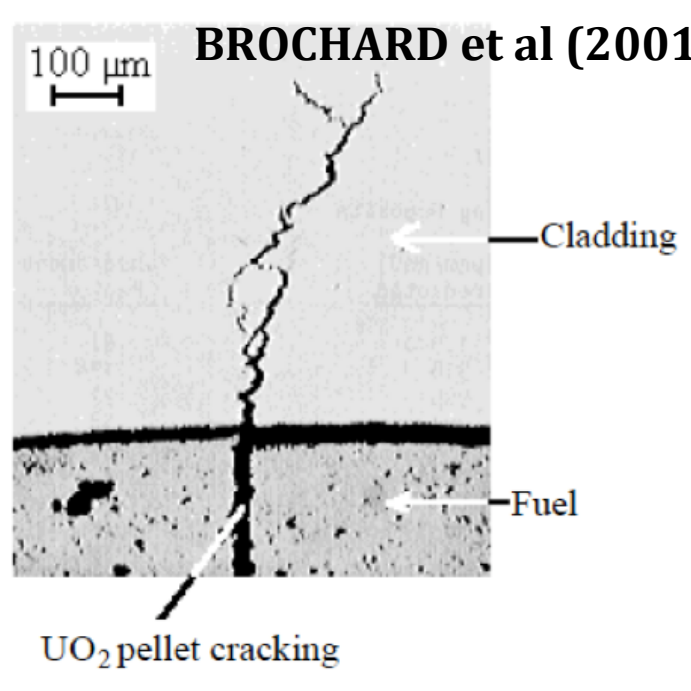

Metals

- Metallic bonding

- No charge transfer

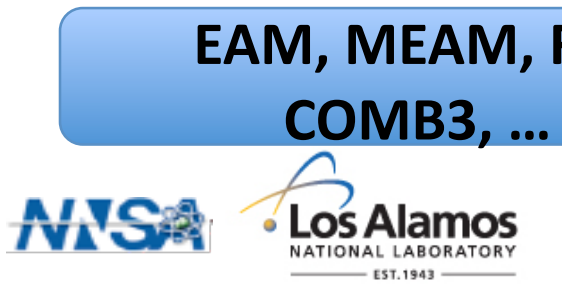

- Corrosion: Fuel-cladding interactions ODS Cladding

- Oxide layer growth and Properties

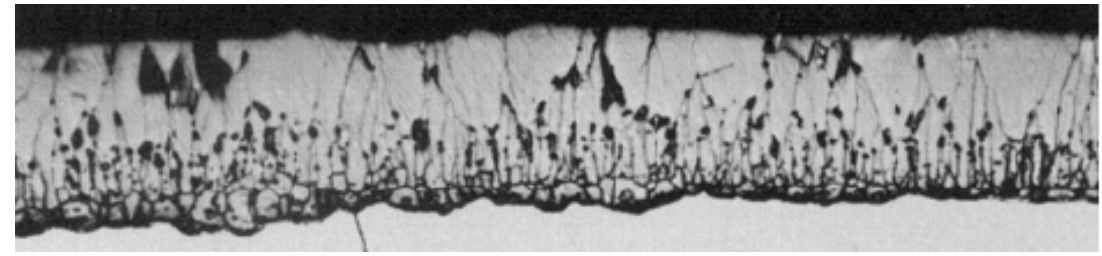

NiO scale on Ni:

Rhines \& Wolf 1970

Mitchell \& Hirth 2008

Ceramics

Covalent

- Angular bonding

- No charge transfer

Tersoff, ReaxFF, COMB, BETM, ...
- Ionic bonding

- Charge transfer controlled heuristically, no physical basis

ReaxFF, COMB, QTPIE, QEq, BETM, ... 


\section{Motivation: STEM-EELS: $\mathrm{Fe}-\mathrm{TiO}_{2}$}

\section{Iron influences titanium valence states near interface}

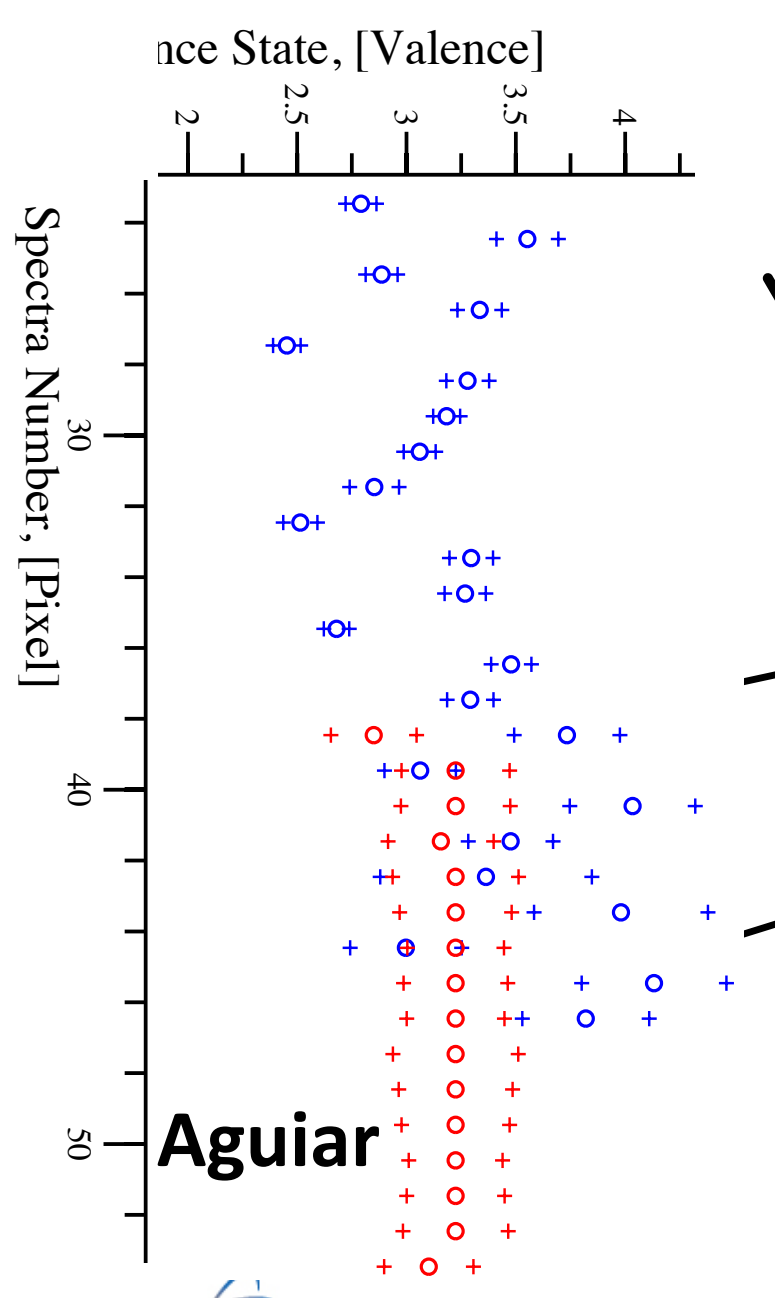

NNGAa TLosAlamos

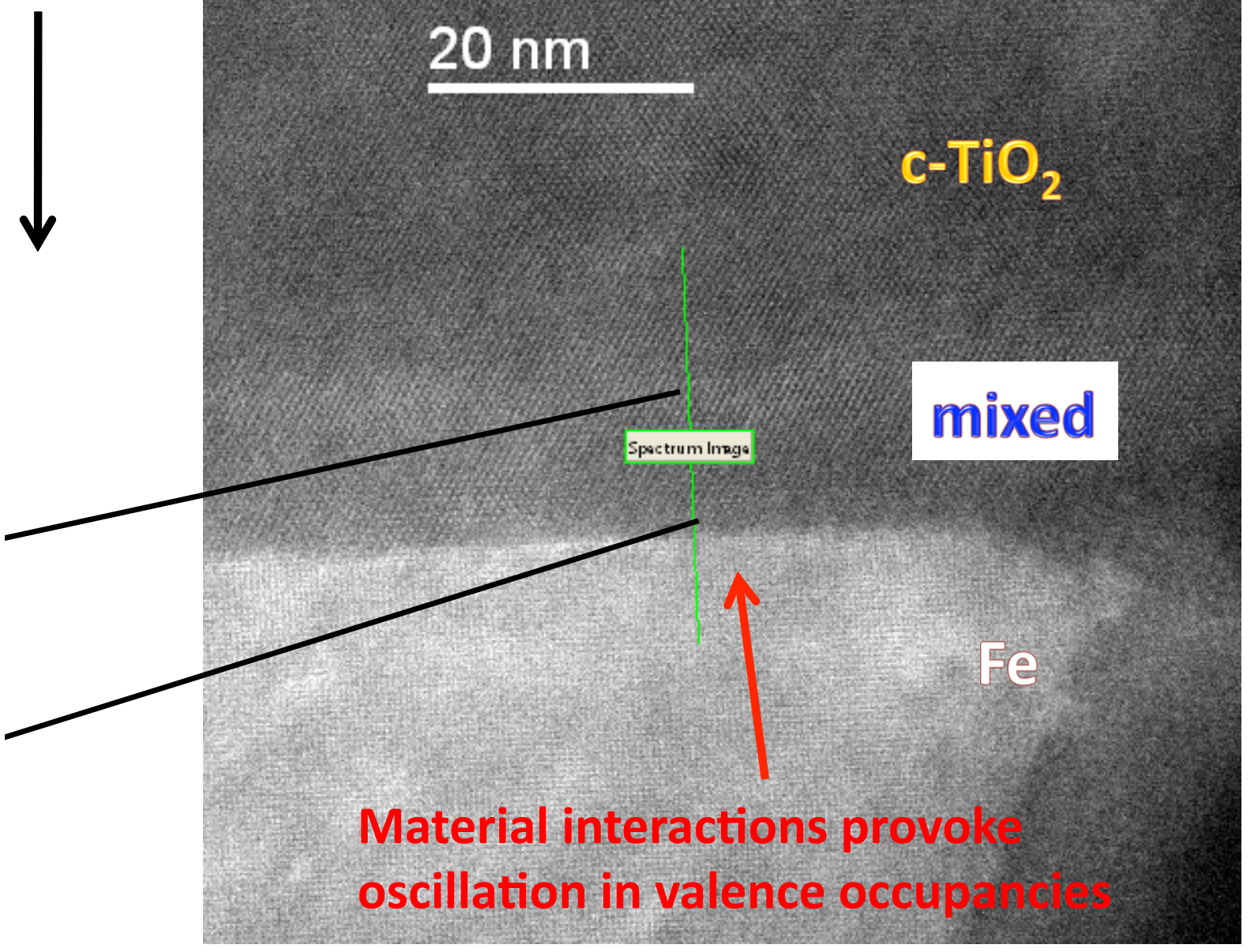

Approach: Model valence states directly

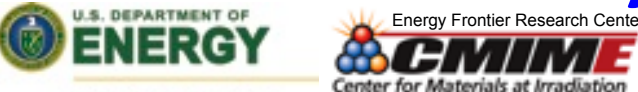




\section{Many $\mathrm{e}^{-}$Hamiltonians in Fragments}

$\hat{H}_{\mathrm{el}} \Psi_{i}=\sum_{\mathrm{A}}\left(\hat{H}_{\mathrm{A}}\left(\boldsymbol{Z}_{\mathrm{A}}\right)+1 / 2 \sum_{\mathrm{B} \neq \mathrm{A}} \hat{\boldsymbol{V}}_{\mathrm{AB}}\left(\boldsymbol{Z}_{\mathrm{A}}, \boldsymbol{Z}_{\mathrm{B}}\right)\right) \psi_{i}$

- Objects defined by Hamiltonians

- "Atom-in-Molecule" Hamiltonian well-known

- Moffitt (1951)

- Always assigned number of electrons $\mathbf{N}_{A}$ equal to nuclear charge $Z_{A}$

- Makes sense physically, but " $\mathrm{N}_{\mathrm{A}}=\mathrm{Z}_{\mathrm{A}}$ " assumption problematic $|A B\rangle=C_{0}\left|A^{0} B^{0}\right\rangle+C_{+}\left|A^{+} B^{-}\right\rangle+C_{-}\left|A^{-} B^{+}\right\rangle$ Pauling, 1929, Slater 1931, Mulliken, 1934 


\section{Fragment Hamiltonian (FH) Model as a Model Hamiltonian}

Fragment:

$E_{\mathrm{A}}=\operatorname{tr} \mathrm{H}_{\mathrm{A}} \Gamma_{\mathrm{A}}\left(C_{+}, C_{0}, C_{-}\right)$

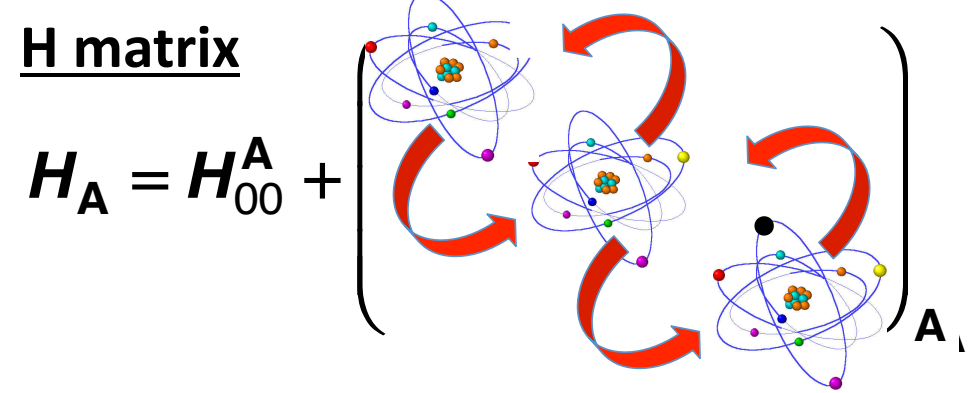

Fragment - Fragment:

$V_{\mathrm{AB}}=V_{00}^{\mathrm{AB}}+\left(\begin{array}{ccc}\boldsymbol{v}_{++}-\boldsymbol{V}_{00} & \boldsymbol{v}_{0+} & \boldsymbol{V}_{+-} \\ \dagger & 0 & \boldsymbol{V}_{0-} \\ \dagger & \dagger & \boldsymbol{V}_{--}-\boldsymbol{V}_{00}\end{array}\right)_{\mathrm{AB}}$

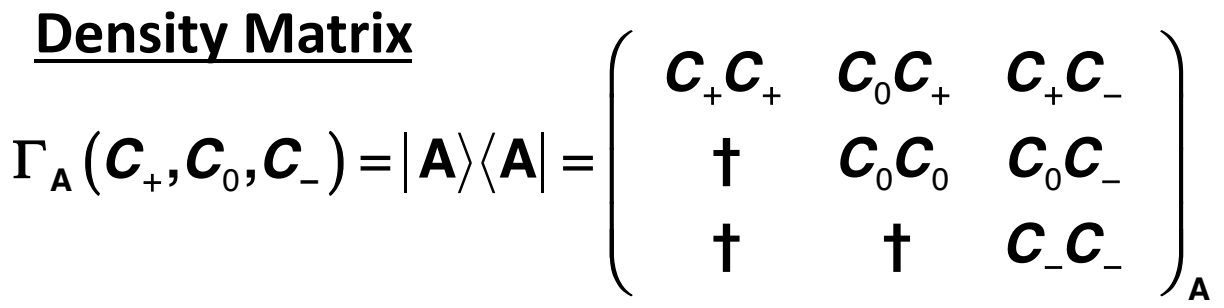

Recovers energy scales: Ionization, Attachment,

$\Gamma_{\mathrm{AB}}\left(\boldsymbol{C}_{+}, \boldsymbol{C}_{0}, \boldsymbol{C}_{-}\right)=(|\mathrm{B}\rangle\langle\mathrm{A}|+| \mathrm{A}\rangle\langle\mathrm{B}|) / 2$

\section{States of atoms paramount}




\section{Approach I: Empirical Atomistic Modeling}

$$
\begin{aligned}
& E=\sum_{\mathrm{A}}\left(F_{\mathrm{A}}+\frac{1}{2} \sum_{\mathrm{B} \neq \mathrm{A}} \phi_{\mathrm{AB}}\left(R_{\mathrm{AB}}\right)\right) \\
& F_{\mathrm{A}}^{\text {metal }}=\text { fcn of val. } e^{-} \text {density } \\
& F_{\mathrm{A}}^{\text {ionic }}=\text { polynomial in charge }
\end{aligned}
$$$$
\phi_{A B}^{\text {metal }}=\text { Short }- \text { Range Pair }
$$$$
\phi_{\mathrm{AB}}^{\text {ionic }}=\text { Buckingham + Electrostatic }
$$

- Basic Paradigms the Same: Site Energies + Site-Site Interactions

- Site: Embedding/Fragment/Atom Completely different physical origins for metals and ceramics

- Site-Site: Pair potentials, 3-body terms

- Only ceramics models are "variational"

Derive from common point of view 


\section{Unified Embedding Energy: Background- $\rho$ and charge $q$}

$$
\begin{aligned}
F_{\mathrm{FH}}\left(\bar{\rho}_{+}, \bar{\rho}_{-}\right) & =F_{\mathrm{FH}}^{(\text {gap })}\left(\bar{\rho}_{+}, \bar{\rho}_{-}\right) \\
& -F_{\mathrm{FH}}^{(\text {hop })}\left(\bar{\rho}_{+}, \bar{\rho}_{-}\right)
\end{aligned}
$$

Two background densities, anion \& cation

$F_{\mathrm{FH}}^{(\text {gap })} \rightarrow$ Gaps, chem. potl., hops

$$
F_{\mathrm{FH}}^{(1 e-h o p)} \rightarrow \quad \text { neutral } \leftrightarrow \text { anion }
$$$$
\text { neutral } \leftrightarrow \text { cation }
$$

- Embedding energies

$$
\boldsymbol{q} \equiv \frac{\bar{\rho}_{+}^{2}-\bar{\rho}_{-}^{2}}{1+\bar{\rho}_{+}^{2}+\bar{\rho}_{-}^{2}}
$$

- Mulliken Electronegativity:

$$
\mu=-(\mathcal{I}+\mathcal{E}) / 2
$$

- Fragment Hardness, Local "Hubbard U":

$$
\boldsymbol{U}=\boldsymbol{I}-\mathcal{E}
$$

smv, JCTC, JPCL 2011

$$
F_{\mathrm{F}-\mathrm{S}}(\tau) \sim\left(W_{1 e}^{+}+W_{1 e}^{-}\right) \sqrt{\bar{\rho}} \quad E(q)=E_{0}+\chi q+\eta q^{2}
$$




\section{Embedding for Elements: $\mathbf{N i}$}

$$
\begin{aligned}
& F_{\mathrm{FH}}\left(\bar{\rho}_{+}=\bar{\rho}_{-}\right)=F_{\mathrm{FH}}^{(\text {gap })}\left(\bar{\rho}_{+}=\bar{\rho}_{-}\right)-F_{\mathrm{FH}}^{(\text {hop })}\left(\bar{\rho}_{+}=\bar{\rho}_{-}\right) \\
& \text {Gap }+2 e^{-} \\
& 1 e^{-}
\end{aligned}
$$

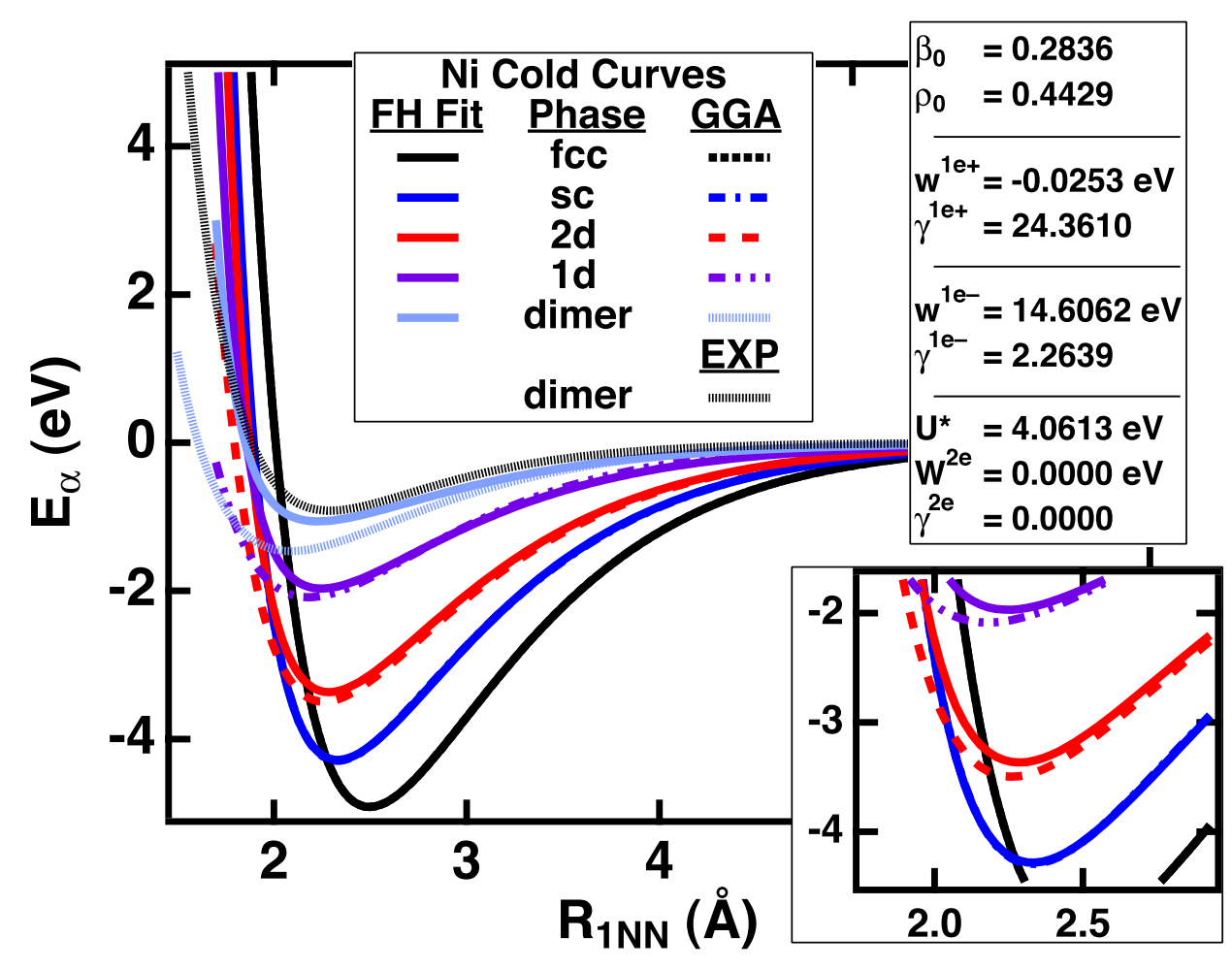

NISSA: LOSAlamos
- Get 3d structures

- Have difficulty with $2 \mathrm{~d}$ and $1 \mathrm{~d}$

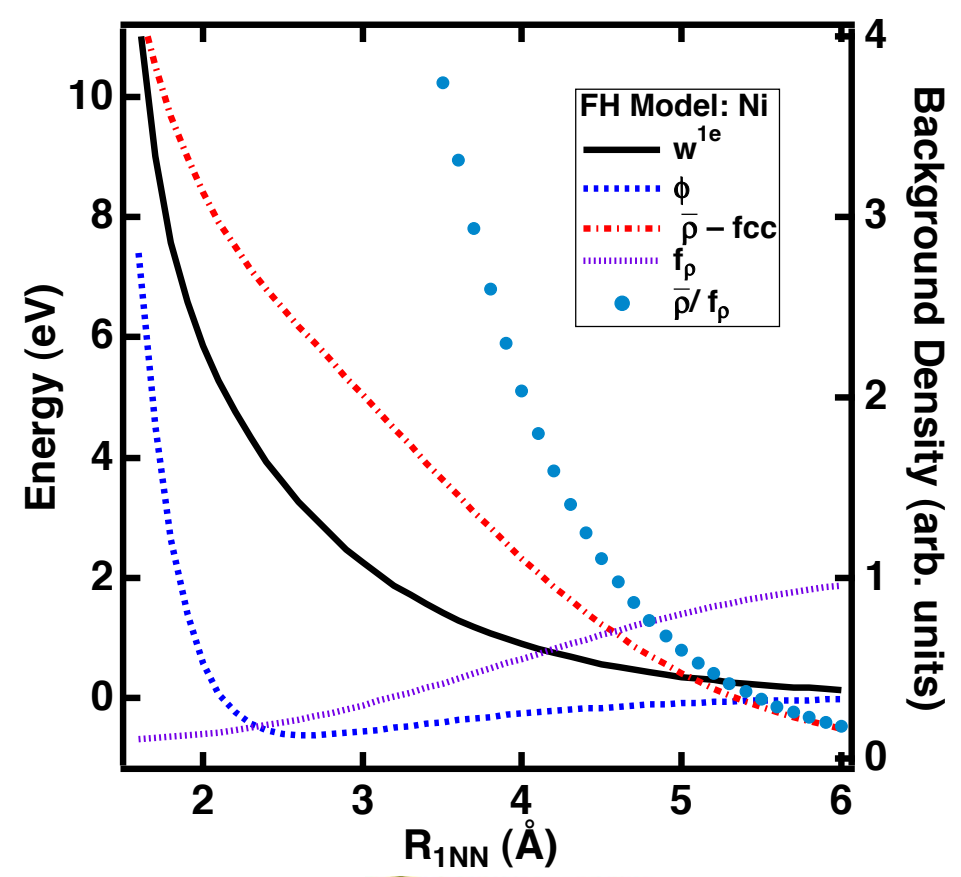

(ㄱ) ËENERGY

Office of Science 


\section{FH Ni: Metallic Character}

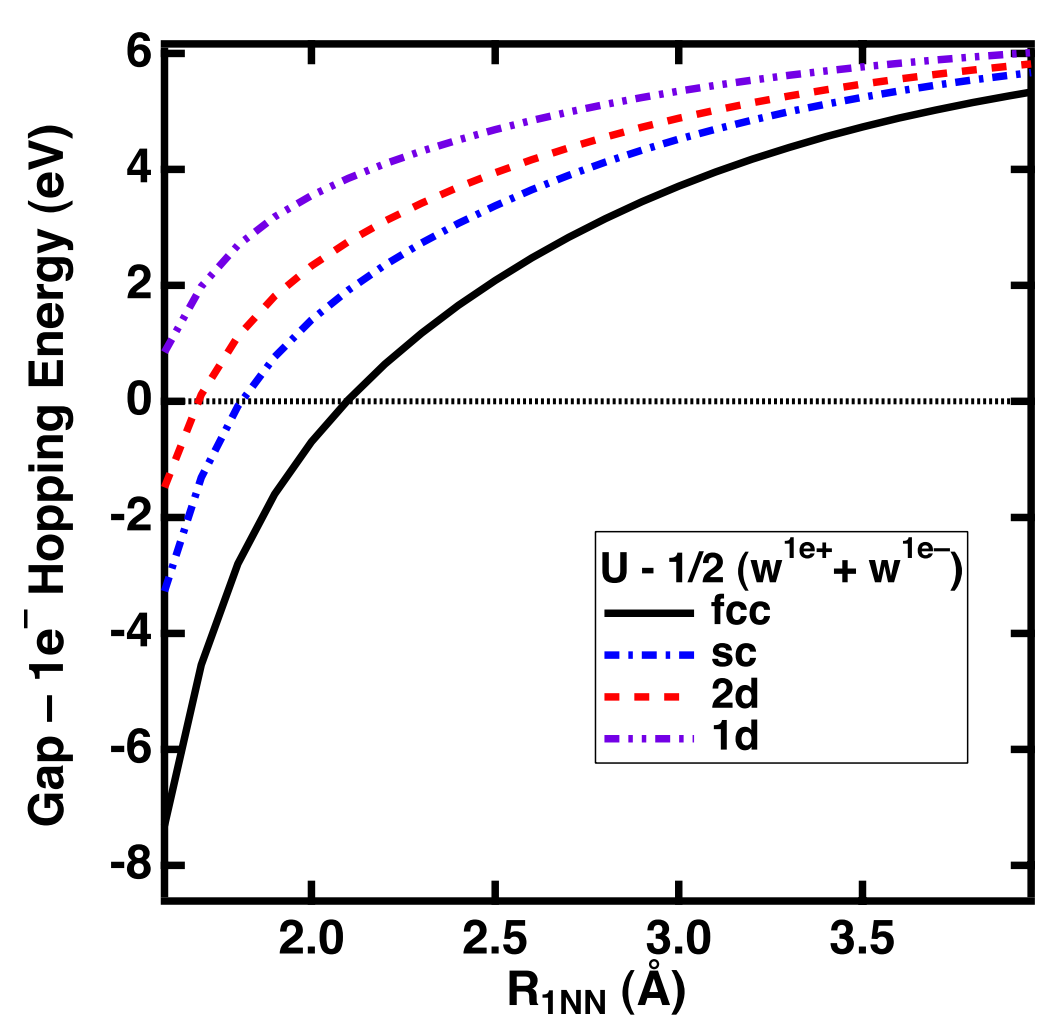

- Keep some notion of concept from solid state physics

- Qualitative match

- Still need other properties to conclude material is "metallic"

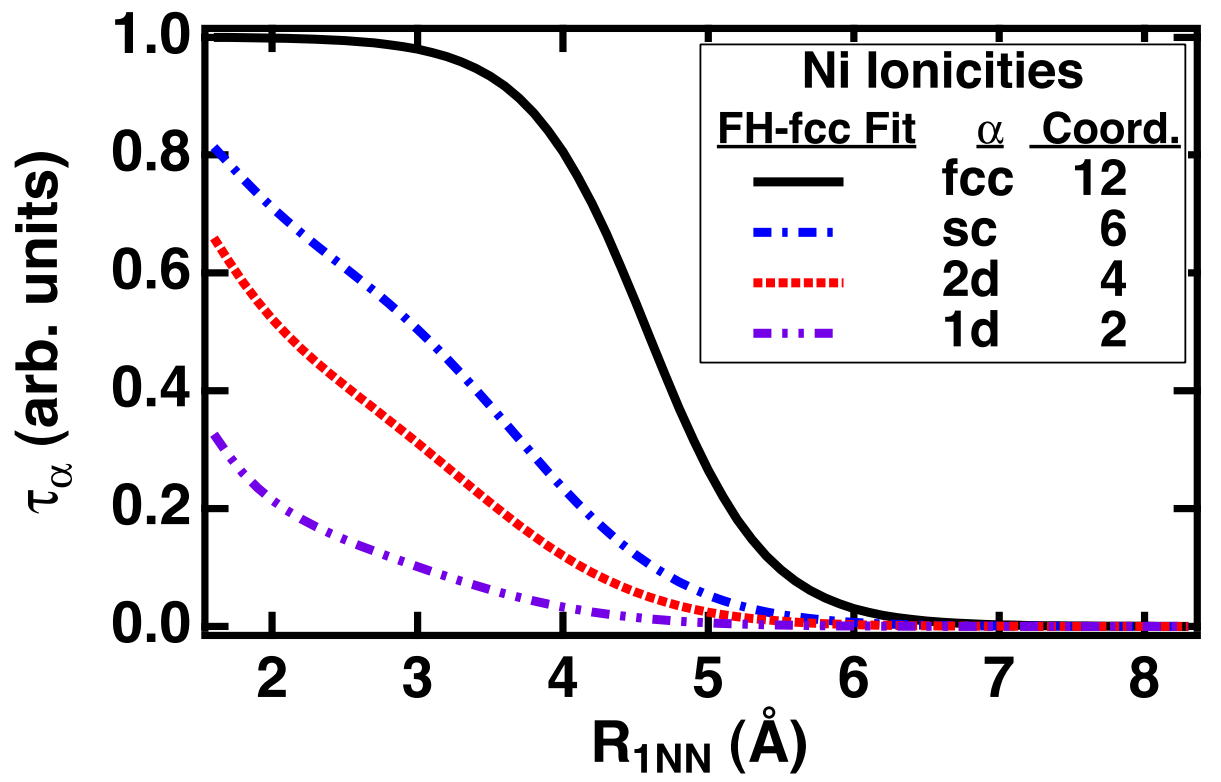

N'SA: LosAlamos

(i) ENERGY 


\section{Ni Elastic Constants, Defects, Surfaces}

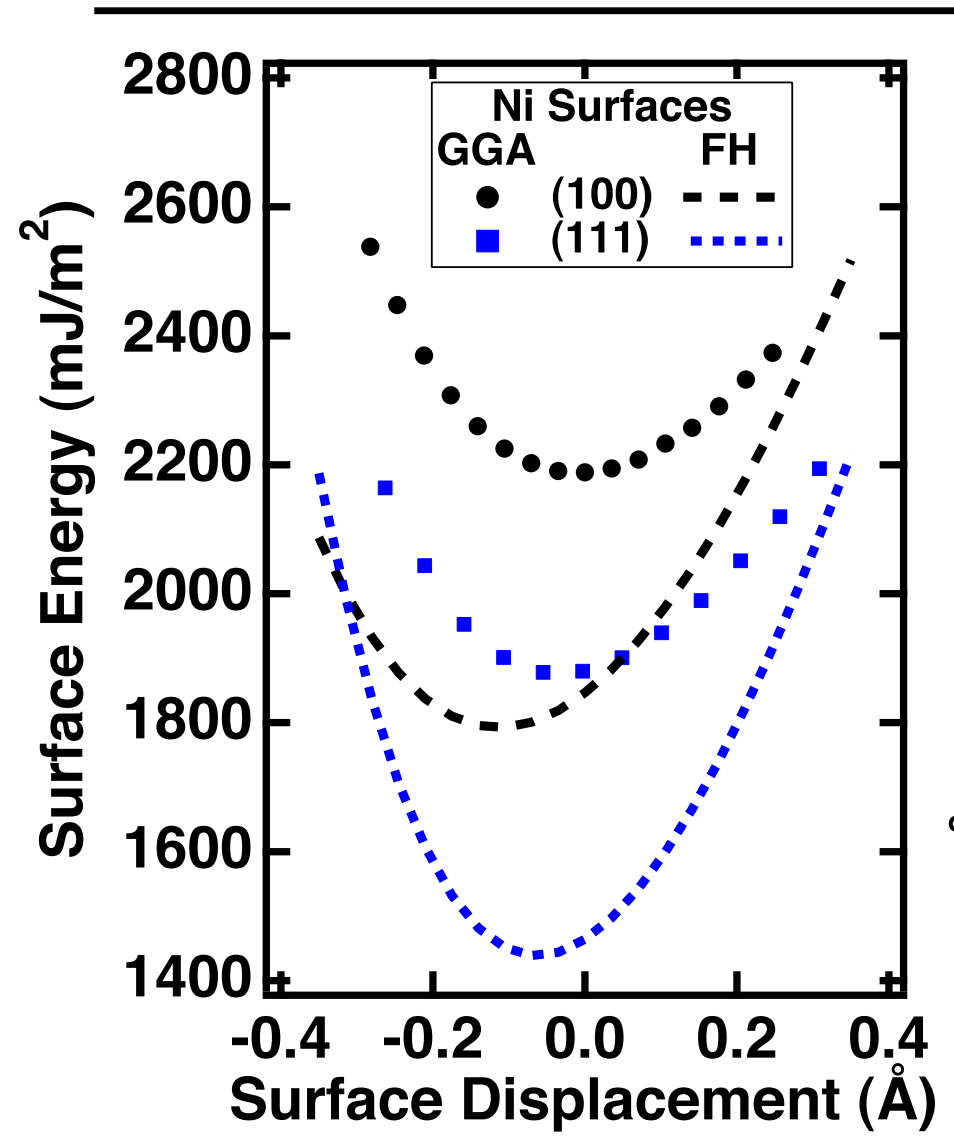

- Same theme: Get 3d structures Have difficulty with $\mathbf{2 d}$ and $\mathbf{1 d}$

- $\mathrm{C}_{11}, \mathrm{C}_{44}$ good; $\mathrm{C}_{12}$ off as expected - Vacancy formation energy good

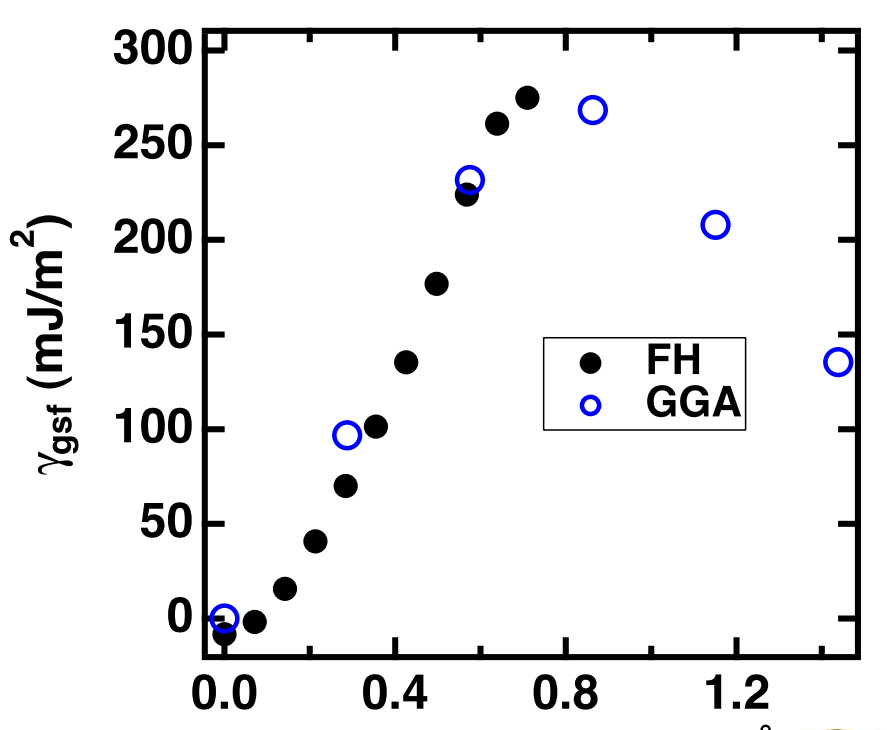

Josh

NISGA: LOSAlamos 


\section{Lattice Distortions, Phase Changes, Defects}

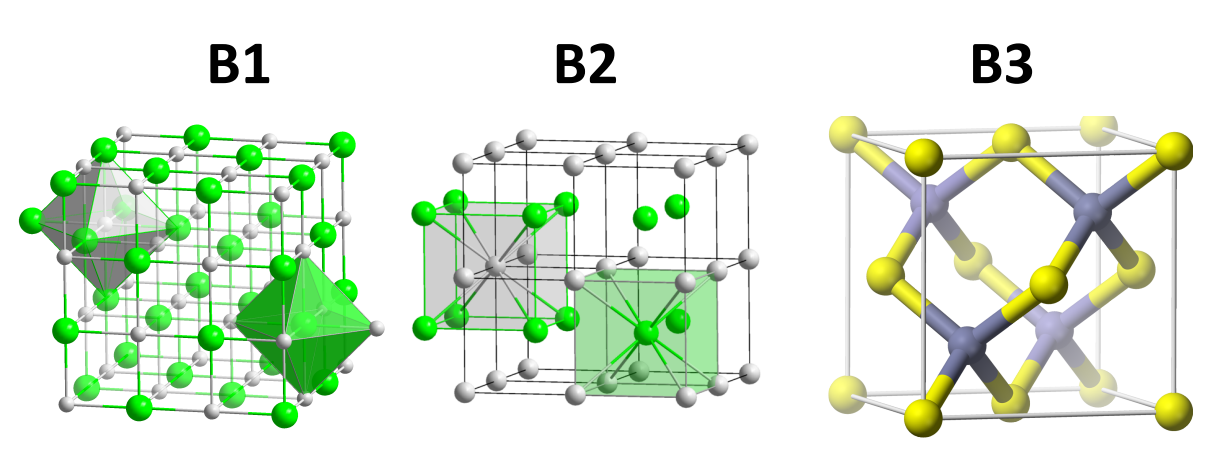

- FH model sensitive to different crystal structures (neighbors, deformations), similar to MEAM, NMEX (Taylor), A-EAM, COMBS
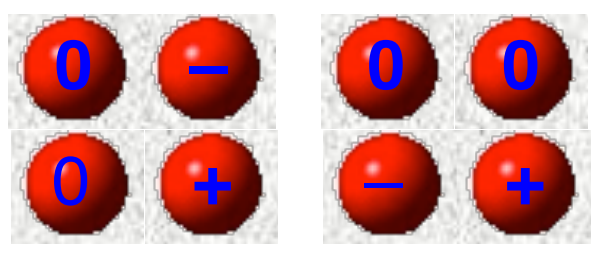

Three-body

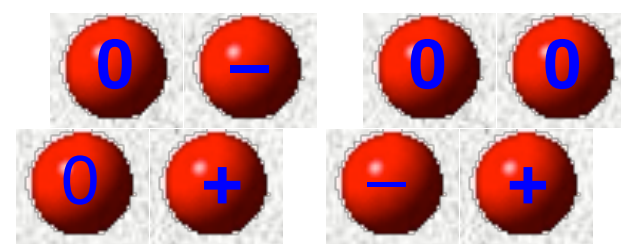

shape-dependent

$$
\boldsymbol{U}_{\mathrm{A}}^{*}\left(\boldsymbol{h}_{\mathrm{BAB}^{\prime}}^{+}, \boldsymbol{h}_{\mathrm{BAB}^{\prime}}^{-}, \tau_{\mathrm{B}}, \tau_{\mathrm{B}^{\prime}}\right)
$$

Need dimensional dependence, shape changes

$$
W_{\mathrm{A}}^{1 \mathrm{ee}} \sim \frac{L_{\mathrm{A}^{+}}}{\sqrt{L_{\mathrm{A}^{+}}}} w_{\mathrm{A}}^{1 \mathrm{e}}=\sqrt{L_{\mathrm{A}^{+}}} w_{\mathrm{A}}^{1 \mathrm{e}}
$$




\section{First Principles Route to Ionicity}

- Decompose density from e.g. DFT

- Fit to form from FH theory

- Good agreement with Bader analysis for charge q

$$
\rho_{\mathrm{A}}=\rho_{\mathrm{A}^{0}}+C_{\mathrm{A}^{+}}^{2}\left(\rho_{\mathrm{A}^{+}}-\rho_{\mathrm{A}^{0}}\right)+C_{\mathrm{A}^{-}}^{2}\left(\rho_{\mathrm{A}^{-}}-\rho_{\mathrm{A}^{0}}\right)
$$
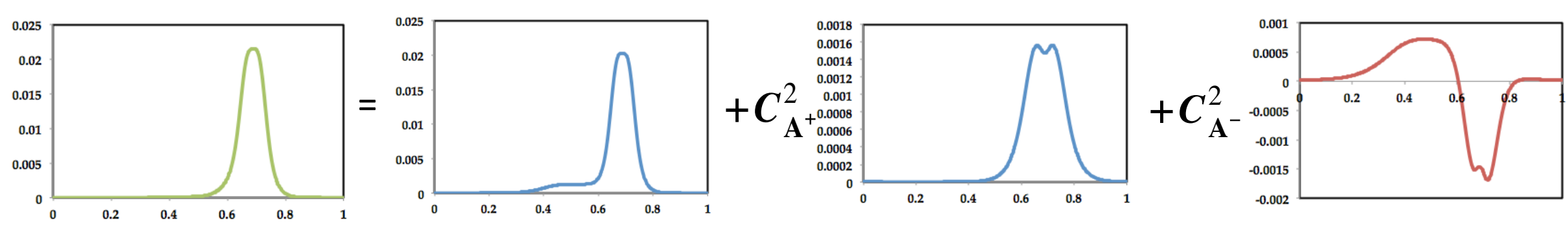

$$
x \rightarrow \quad q_{\mathrm{A}}=C_{\mathrm{A}^{+}}^{2}-C_{\mathrm{A}^{-}}^{2}
$$

$$
\tau_{A}=C_{A+}^{2}+C_{A-}^{2}
$$




\section{Gaps and Metallicity Appear in FH: Be Densities of States}
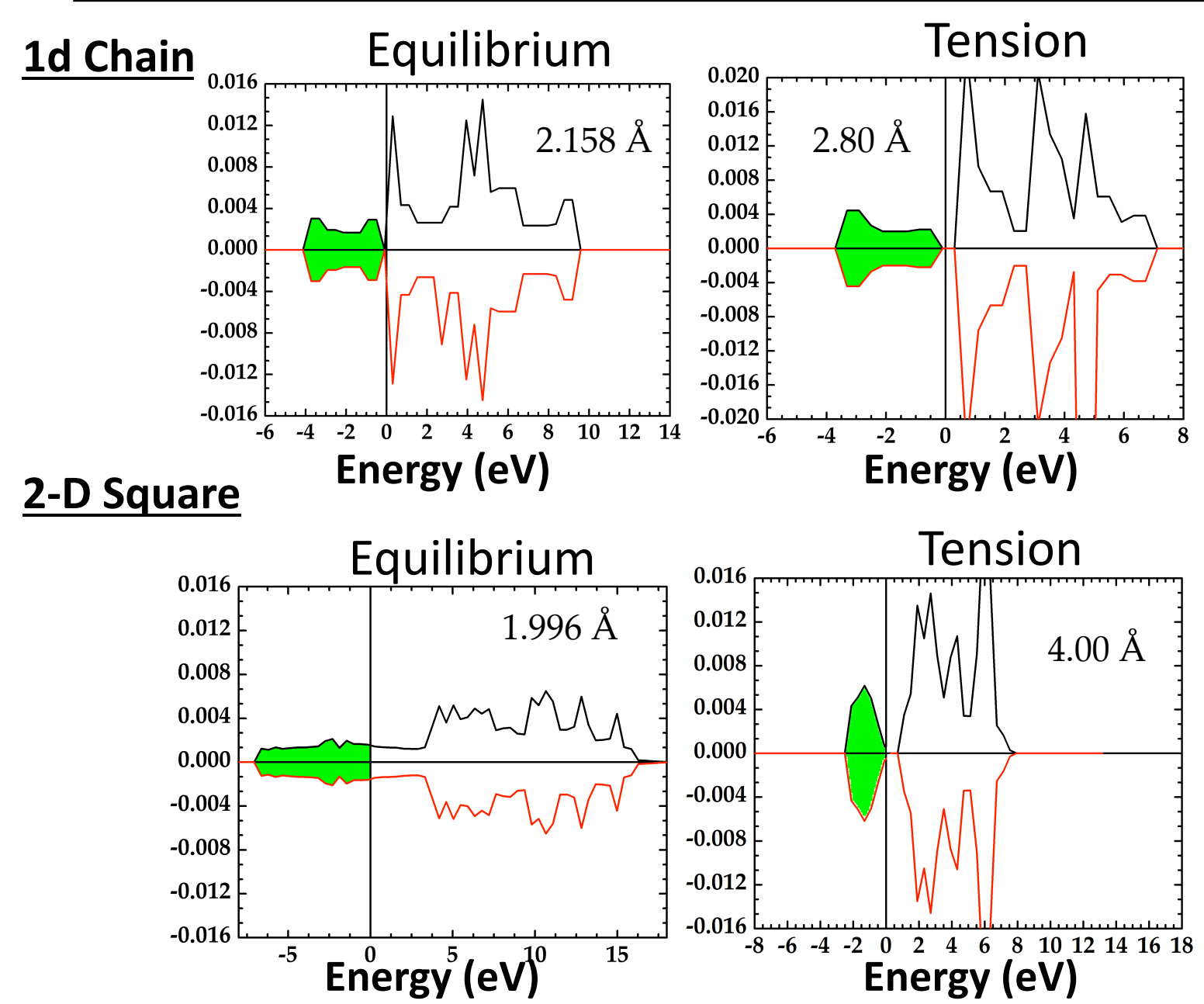

- Gaps open and close in e-structure calculations

- Mirror these essential features in FH model

Pilania 


\section{Approach II (with UNM): Model H}

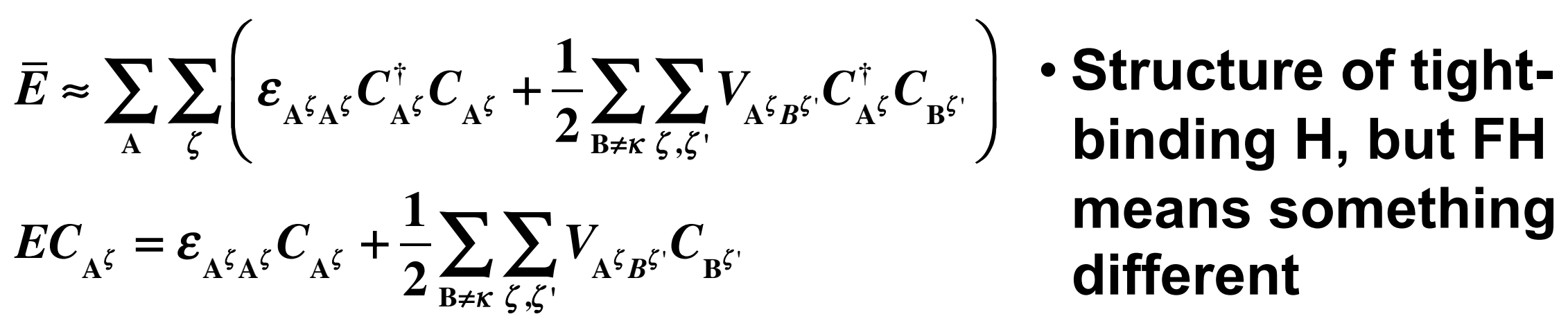

- Green fcn introduces

$\Gamma(z)=-\frac{1}{\pi} \operatorname{Im} G\left(z^{+}\right)$ contributions from the

$\rightarrow \operatorname{tr} \delta\left(z S-\mathbf{H}_{\Gamma}-\mathbf{H}_{*}-V_{\Gamma^{*}}\right) \Gamma$

- Related to vast knowledge, data of electron spectroscopy, electronic structure calculations spectrum of $\mathrm{H}$

- Density of states (energy) $\Gamma(z)$ observable and computable "Spectral Density Matrix"

- Not used in typical atomic-scale models NISAat toosalamos 


\section{Model Hamiltonian Form: 1D, $\infty$ Chain}

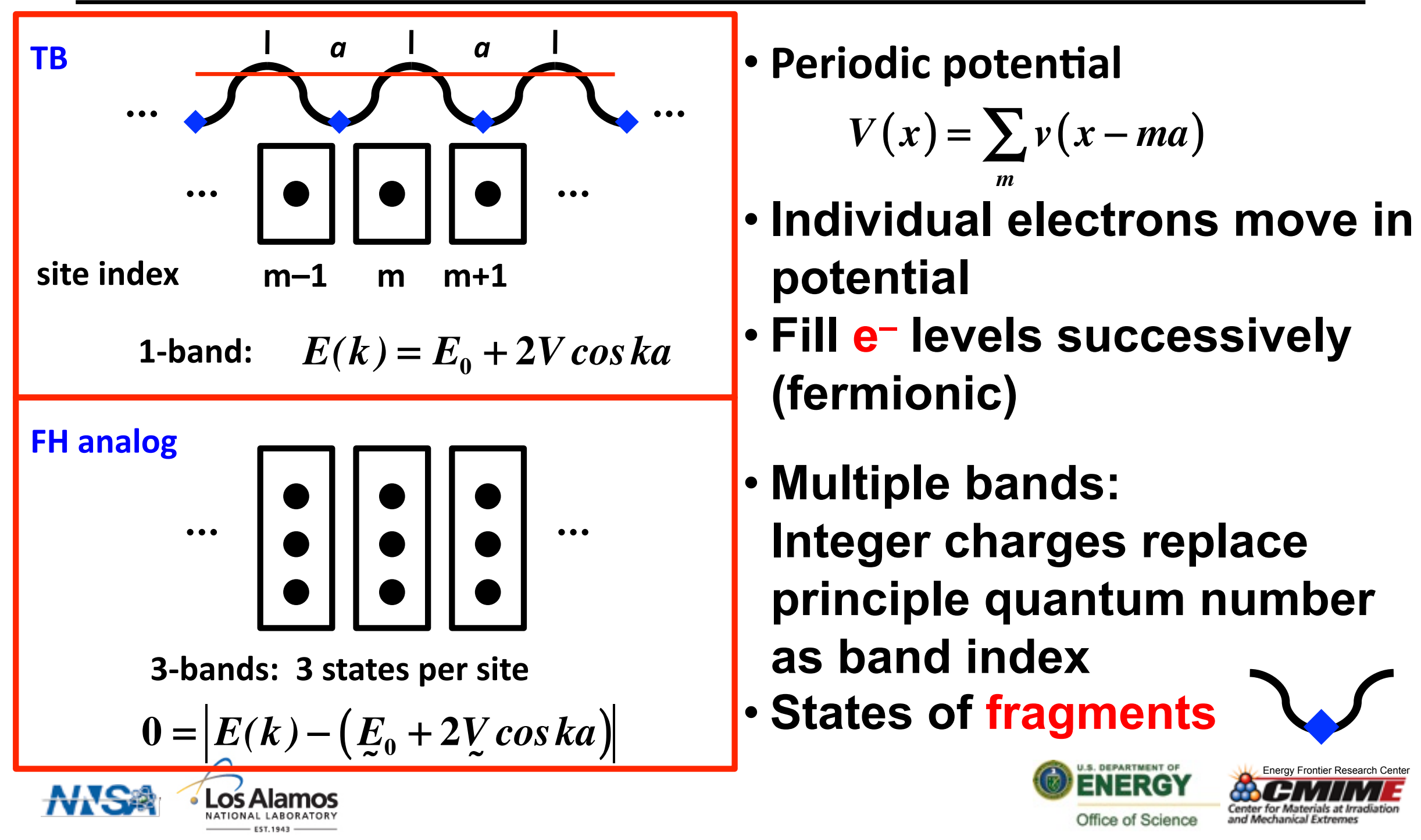




\section{FH Model for 1D, Periodic, $\infty$ Chain: Gap Closure}

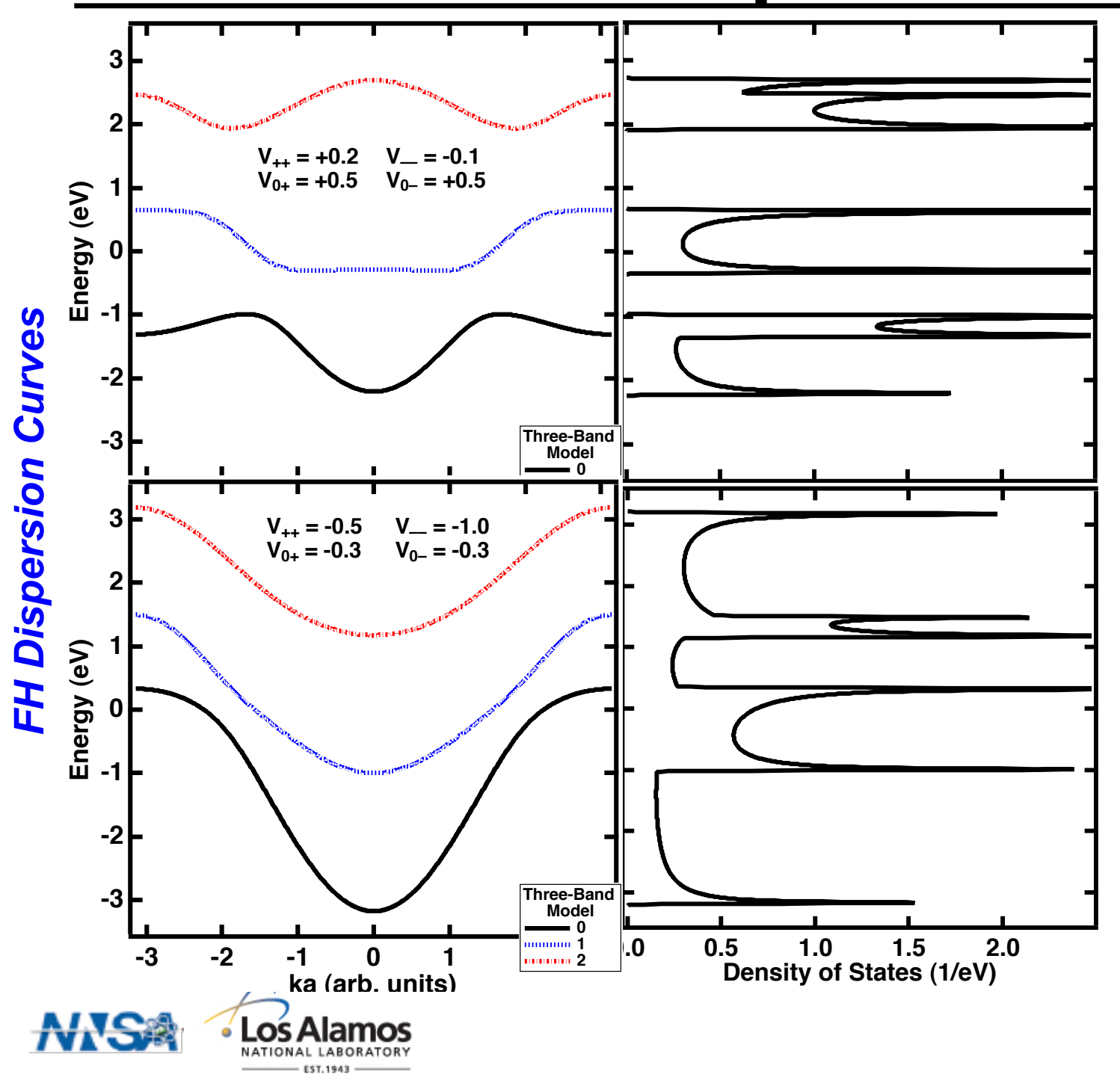

For 1D chain

- Multiband TB: metal or insulator depending on filling

- Hubbard model: No gap closure unless $U \rightarrow 0$ cf Lieb \& Wu, 1968

- Atomistic FH model: Gaps open, close with "local $U$ " and hopping

$$
\begin{array}{ll}
I=2 & \mathrm{~V}_{00}=-0.5 \\
\mathcal{E}=1 & \mathrm{~V}_{+-}=-0.0
\end{array}
$$

FH DOS 


\section{Near Future:}

\section{Link FH Model \& Electron Spectroscopy}

$$
\begin{aligned}
|A \boldsymbol{B}\rangle & =\boldsymbol{C}_{0}\left|\boldsymbol{A}^{0} \boldsymbol{B}^{0}\right\rangle \\
& +C_{+}\left|A^{+} \boldsymbol{B}^{-}\right\rangle \\
& +C_{-}\left|A^{-} \boldsymbol{B}^{+}\right\rangle
\end{aligned}
$$

Wave fon

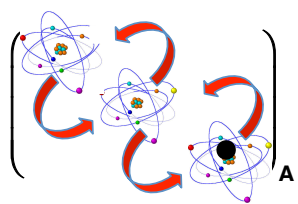

$\underline{H \text { matrix }}$

$T \sim 2 \mathrm{eV}, \Delta \sim 5 \mathrm{eV}$, $U_{d d} \sim 8 \mathrm{eV}$

N'SA: LosAlamos
FH \& PES (from Hüfner, 1994)

$$
\left|\mathbf{N i}^{+2} \mathbf{O}^{-2}\right\rangle,\left|\mathbf{N i}^{+1} \mathbf{O}^{-1}\right\rangle,\left|\mathbf{N i}^{0} \mathbf{O}^{0}\right\rangle
$$

$\mathrm{NiO}$ mimicked by $\mathrm{NiO}_{6}{ }^{10-}$ :

$$
\left|d^{8} \boldsymbol{L}\right\rangle,\left|d^{9} \boldsymbol{L}^{-1}\right\rangle,\left|d^{10} \boldsymbol{L}^{-2}\right\rangle
$$

Matters for structural problems

$$
H_{\mathrm{Ni}}+H_{\mathrm{O}}=
$$

$$
\left(\begin{array}{ccc}
0 & T \sqrt{2} & 0 \\
T \sqrt{2} & \Delta & T \sqrt{2} \\
0 & T \sqrt{2} & 2 \Delta+U_{d d}
\end{array}\right)_{d L}
$$

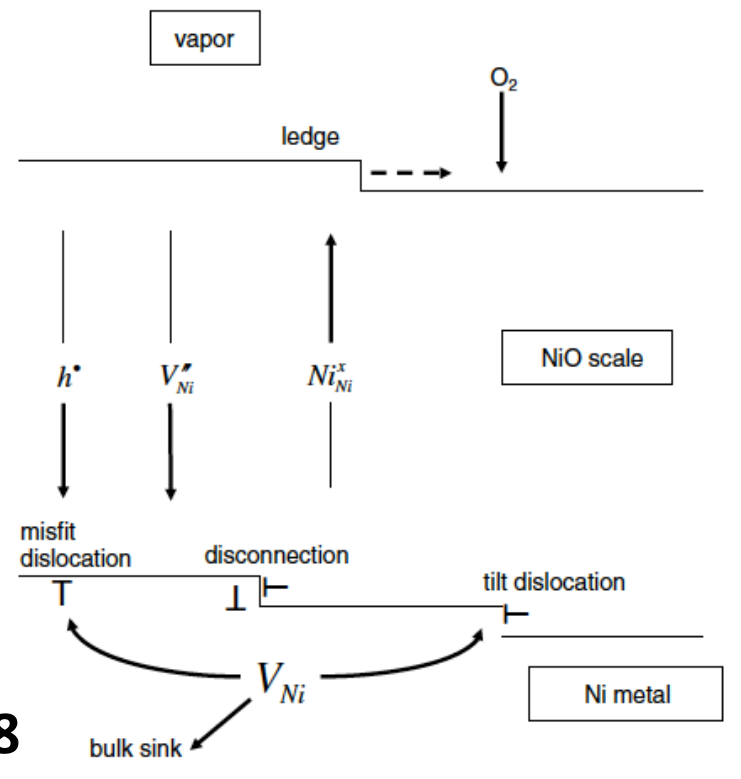

Mitchell \& Hirth, 2008 Model of scale growth 


\section{Other Applications: Charge Separation}

Time Evolution in Composite Materials Electromechanics Grand Challenge

Plasma-Material Interactions

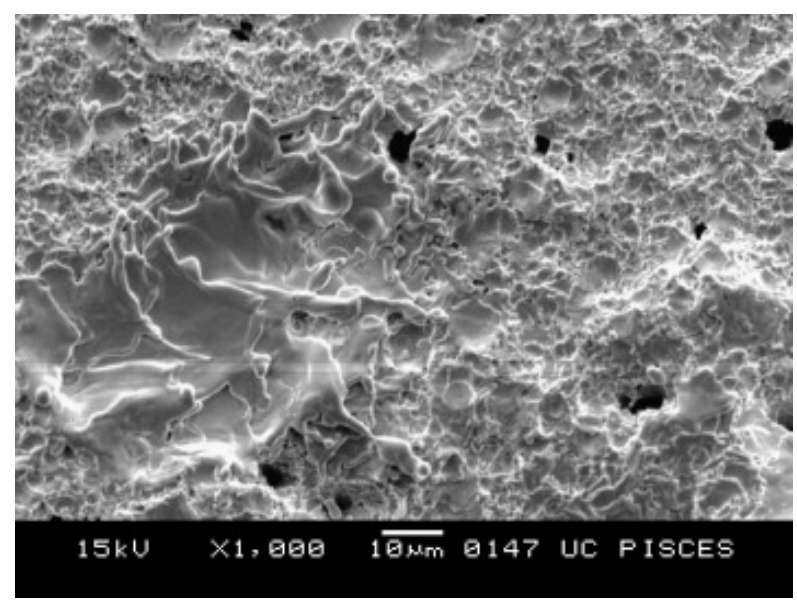

Umstadter, Doerner, Tynan 2009

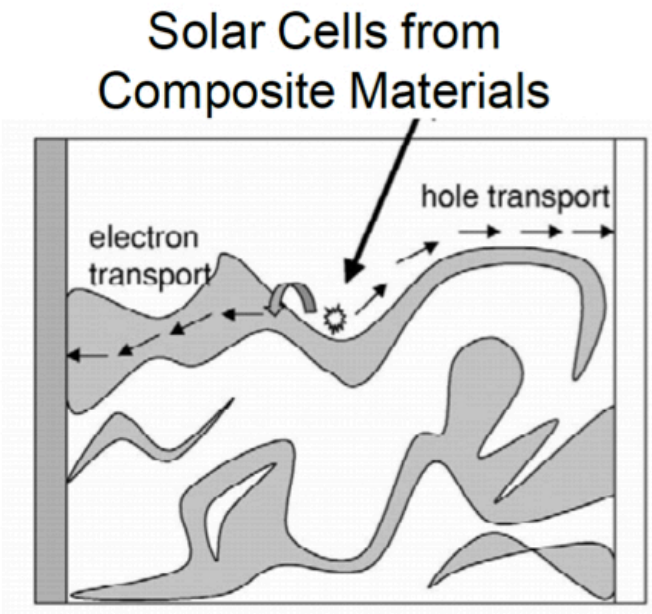

Charge separation at interfaces
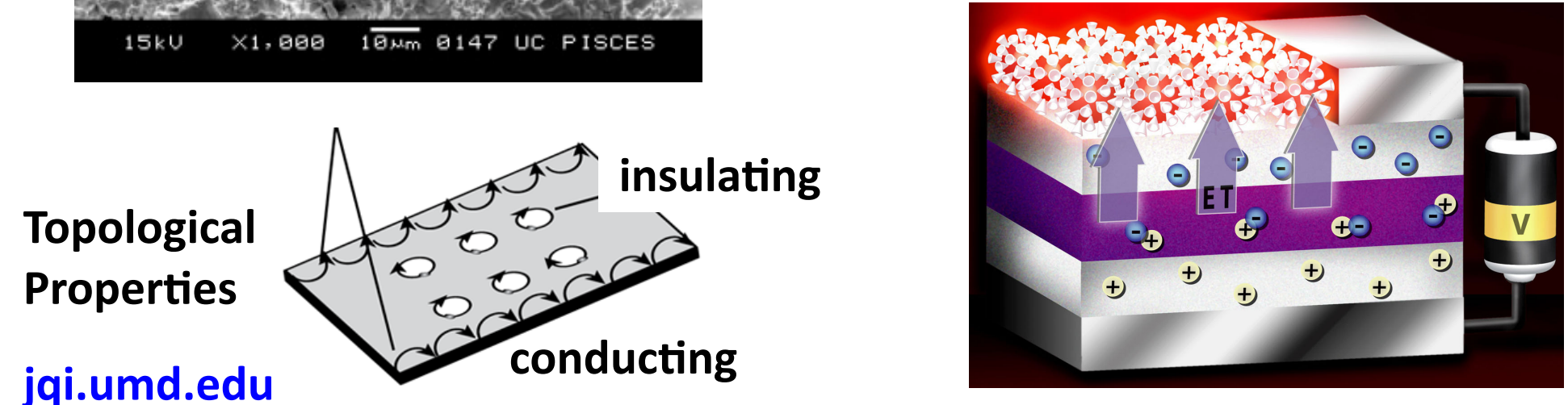

Achermann

et al. 2004 


\section{Summary: FH Model Potentials}

- FH Model for atomic scale

- States of ATOMs, rather than states of $\mathrm{e}^{-} \mathrm{s}$

- Unify models for oxides and metals

- Ni model developed, applied to surfaces, stacking fault E, paper about to be submitted

- Introduce local Hubbard energy or chemical hardness

- Some connection to 3-band tight-binding model

- Gaps and gap closure in an atomistic model

- Apply FH model to NiO based on $\mathrm{Ni}, \mathrm{O}$ 


\section{References}

[1] RP Iczkowski and JL Margrave, J Am Chem Soc 83, 3547 (1961).

[2] HO Pritchard and FH Sumner, Proc Roy Soc, London A 235, 136 (1956).

[3] JP Perdew, RG Parr, M Levy, JL Balduz, Jr, Phys Rev Lett 49, 1691 (1982).

[4] SM Valone and SR Atlas, J Chem Phys 120, 7262 (2004).

[5] SM Valone, J Li, and S Jindal, IJQC 108, 1452 (2008).

[6] RP Feynman, Statistical Mechanics: A Set of Lectures, (AddisonWesley, Reading MA, 1998), Ch. 2

[7] SM Valone, J Chem Theory Comput 7, 2253 (2011)

[8] SM Valone, J Phys Chem Lett 2, 2618 (2011) 
1. 25-30 minute talks (leave 10-15 minutes for discussion).

2. State the gap in capability that your project is trying to fill.

3. State the application of the method you are developing to CMIME research (present and future).

4. Describe your approach, highlighting the qualifications of the team working with you.

5. Highlight the "what's new" aspect in your approach as well as capabilities of your method.

6. Give examples of applications of your method to CMIME.

7. Show a list of publications (published or accepted) directly related to this project (Do not show your other CMIME publications that are NOT directly related to this project).

8. Indicate future plans for this method development project. (i) complete and publish work in the current grant period (through July 2014), and (ii) a vision for next 5 years (if there is a call for renewal proposal, what will you propose?).

9. Indicate other applications of your method, e.g., any spin-off projects not funded by CMIME where the method may be used (be specific).

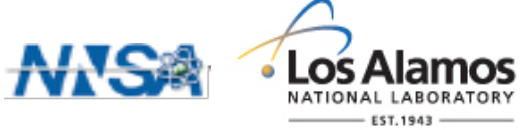




\section{FH Gives Unified Model of Bonding}

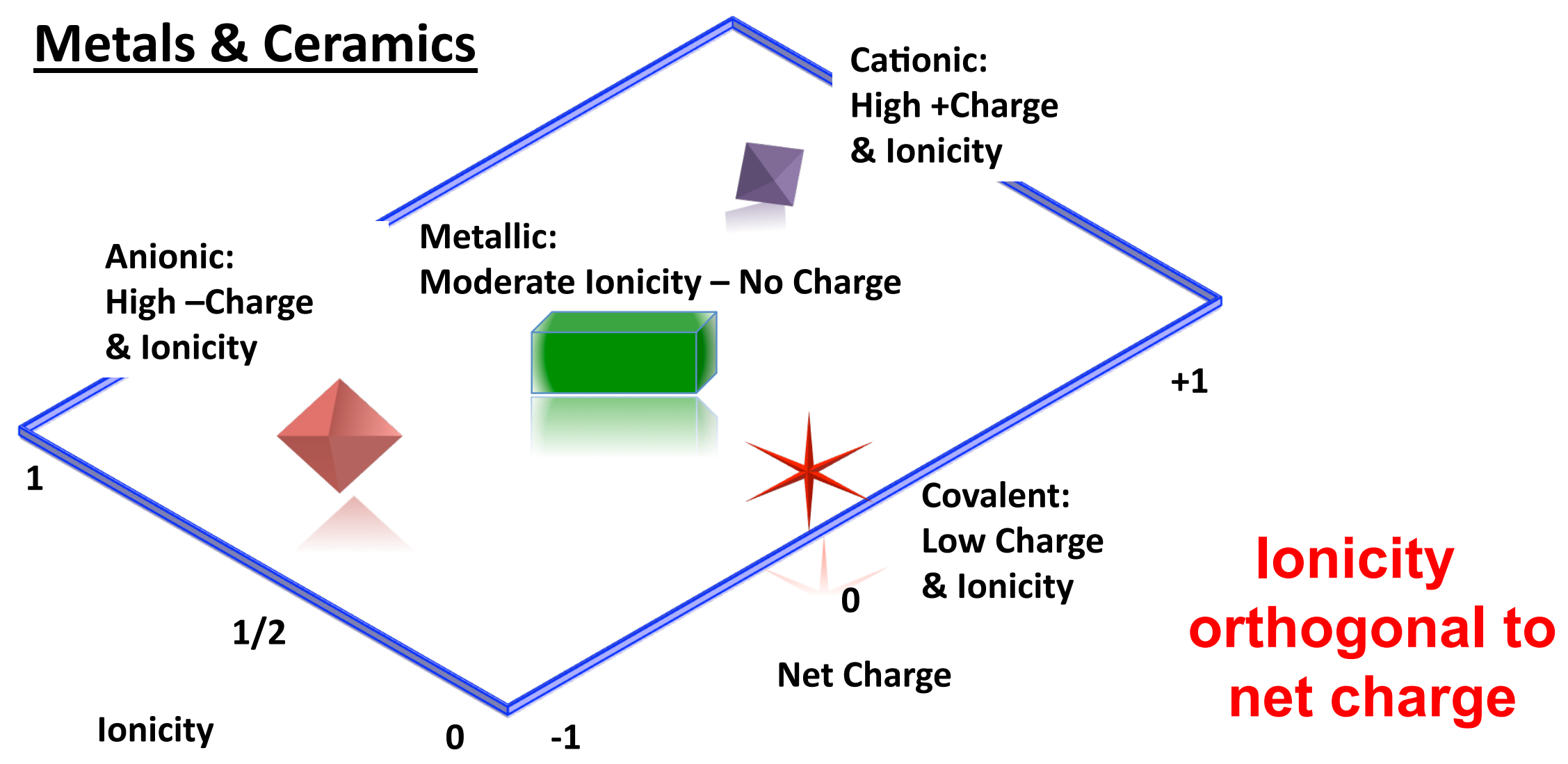




\section{Model Hamiltonian Approach}

Full, Many-electron $\mathbf{H}$

Fragment $\hat{\mathrm{H}}$

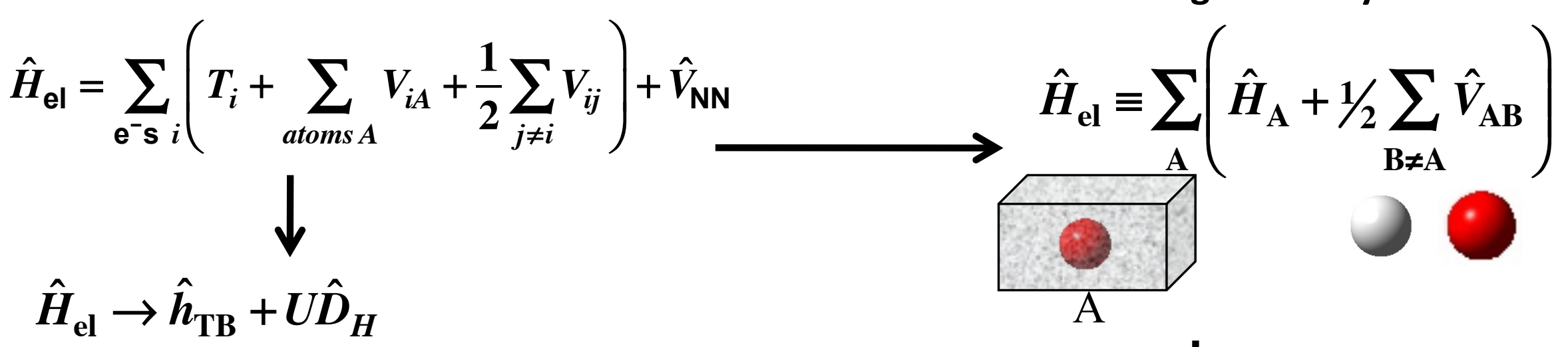

$=-\sum_{R_{\mathrm{A}} \neq R_{\mathrm{B}}, \sigma} t\left(R_{\mathrm{A}}\right.$
$+U \sum_{\mathrm{A}} \hat{n}_{\mathrm{A} \downarrow} \hat{n}_{\mathrm{A} \uparrow}$

Tight-bonding, Hubbard, DFT H's

One-electron granularity

N'SA: LosAlamos 


\section{Missing Element: Charge Fluctuations on Operators}

- Distribute electrons among fragments: Charge fluctuations

- Vary integer number of electrons assigned to fragments $\rightarrow$ Open system

- Superpose states with different numbers of $\mathrm{e}^{-}$on sites

(0) $H_{A}\left(Z_{A}\right) \quad H_{B}\left(Z_{B}\right)$
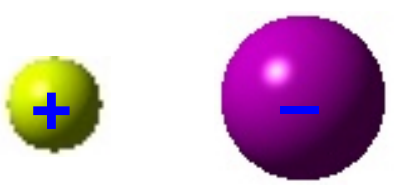

$H_{\mathrm{A}}\left(Z_{\mathrm{A}}-1\right) \quad H_{\mathrm{B}}\left(Z_{\mathrm{B}}+1\right)$

NSGA: TosAlamos

$$
\psi_{00}=\phi_{\mathrm{A}}(1) \phi_{\mathrm{B}}(2)+\phi_{\mathrm{A}}(2) \phi_{\mathrm{B}}(1): \text { Heitler-London }
$$
covalent state

$$
\begin{aligned}
& \psi_{+-}=\phi_{\mathrm{B}}(1) \phi_{\mathrm{B}}(2) \\
& \psi_{-+}=\phi_{\mathrm{A}}(1) \phi_{\mathrm{A}}(2)
\end{aligned}
$$

: ionic states

Note: mean-field/molecular orbital state is an average of $\psi_{00}, \psi_{+_{-}}$and $\psi_{-+}$ 


\section{Metals: Embedded Atom Models}

$$
\begin{gathered}
E=\sum_{\mathrm{A}}\left(F_{E A M}\left(\bar{\rho}_{\mathrm{A}}\right)+\frac{1}{2} \sum_{\mathrm{B} \neq \mathrm{A}} \phi_{\mathrm{AB}}\left(R_{\mathrm{AB}}\right)\right) \\
F_{M E A M}(\bar{\rho})=A \frac{\bar{\rho}}{\rho_{0}} \ln \frac{\bar{\rho}}{\rho_{0}} \\
F_{F-S}(\bar{\rho})=A \sqrt{\frac{\bar{\rho}}{\rho_{0}}}
\end{gathered}
$$

- EAM energy

- Embedding Function

$$
\phi(R)=\frac{2}{Z}\left\{E^{u}(R)-F\left(\bar{\rho}^{u}(R)\right)\right\} \quad \text { - Pair Potential }
$$


FH Density Matrix for Diatomic: Chemical Potential and Charge Equilibration

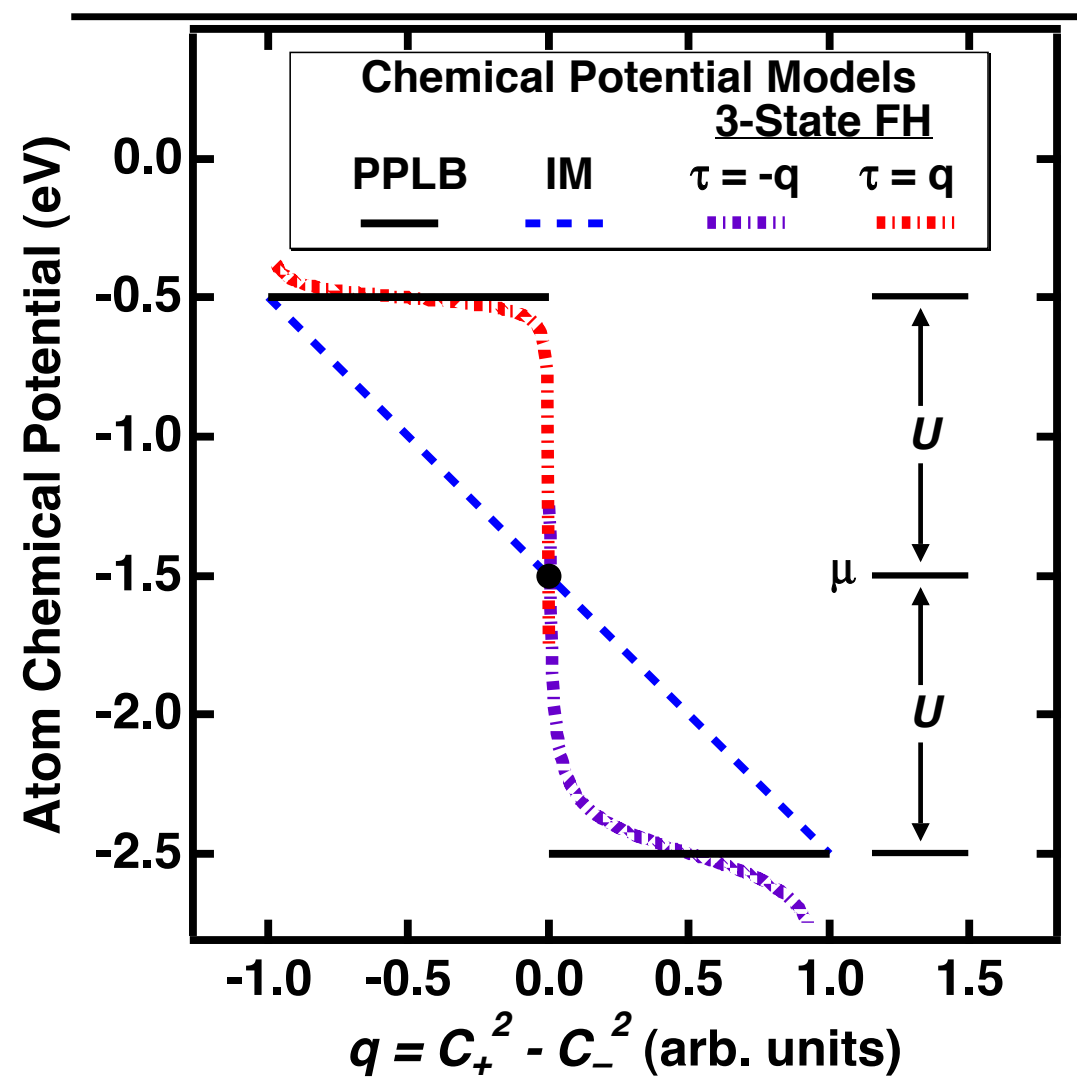

Chemical Potential (dE/dq): Varies greatly among models. PPLB is correct limit for dissociated (gas-phase) atoms. IM is linear.

N'́SG: LOSAlamos

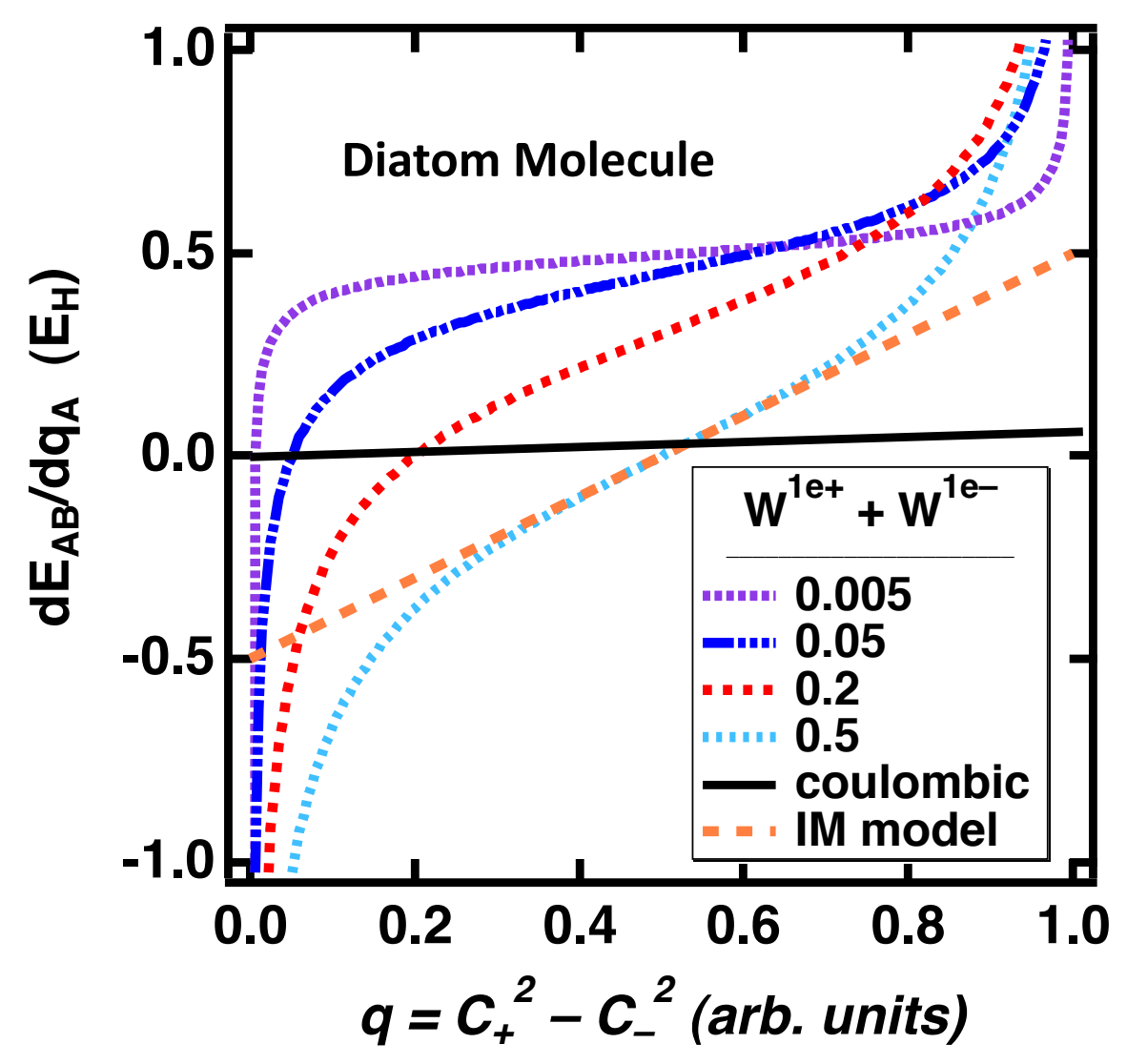

Attenuate charge flows: Crossing of black line with colored curves 


\section{BeO Chain}

Compression

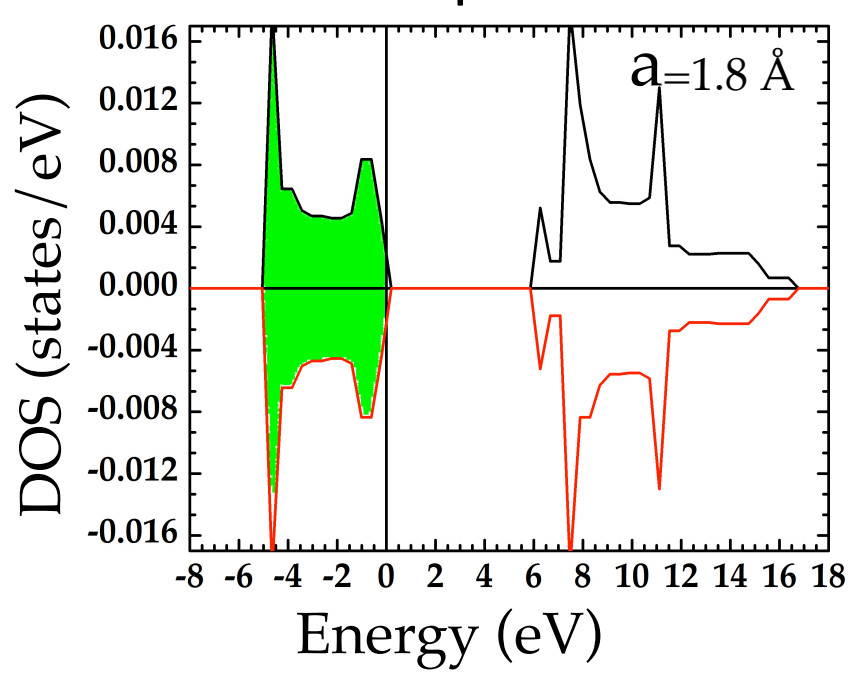

Equilibrium

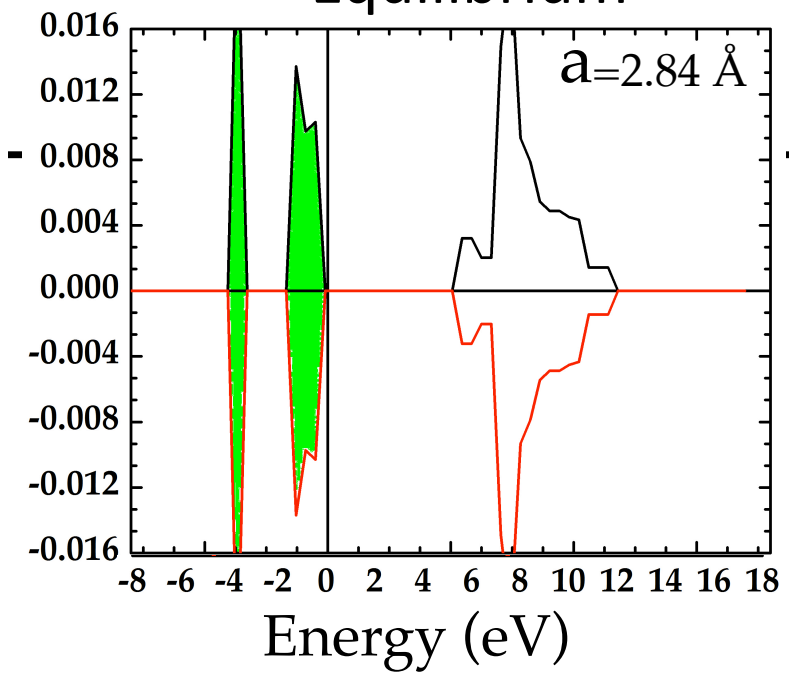

BeO 2-D Square Lattice

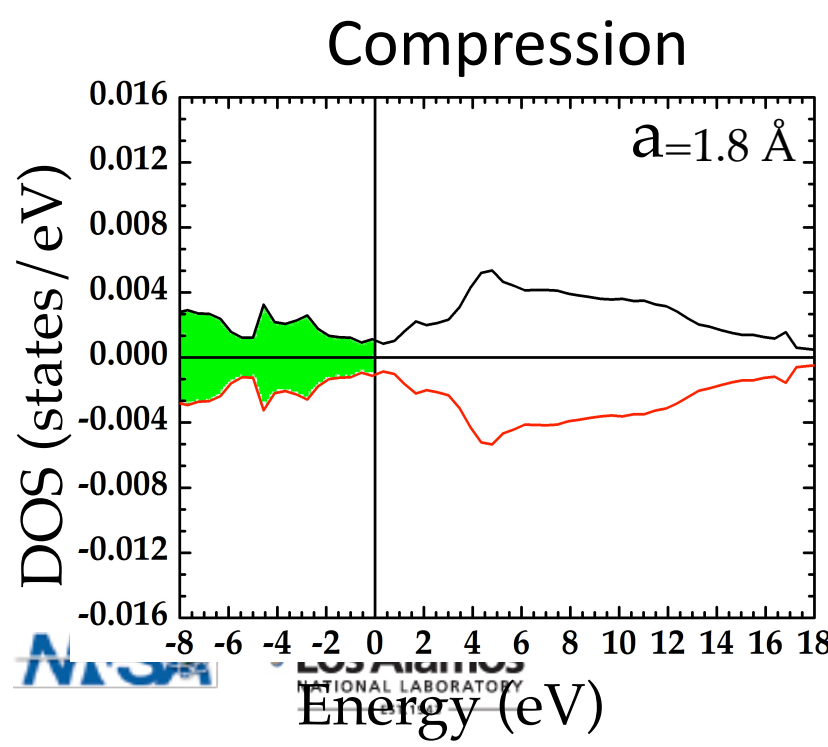

Equilibrium

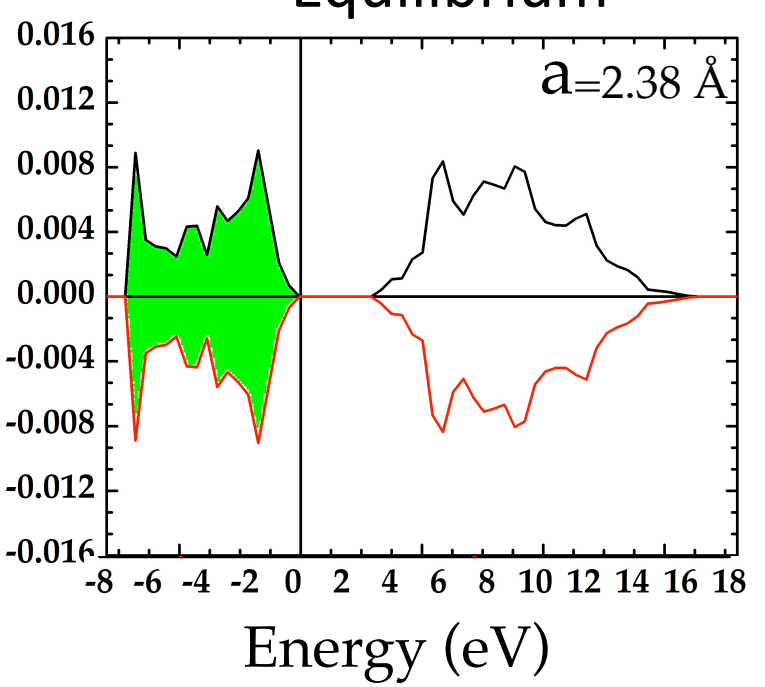

Tension

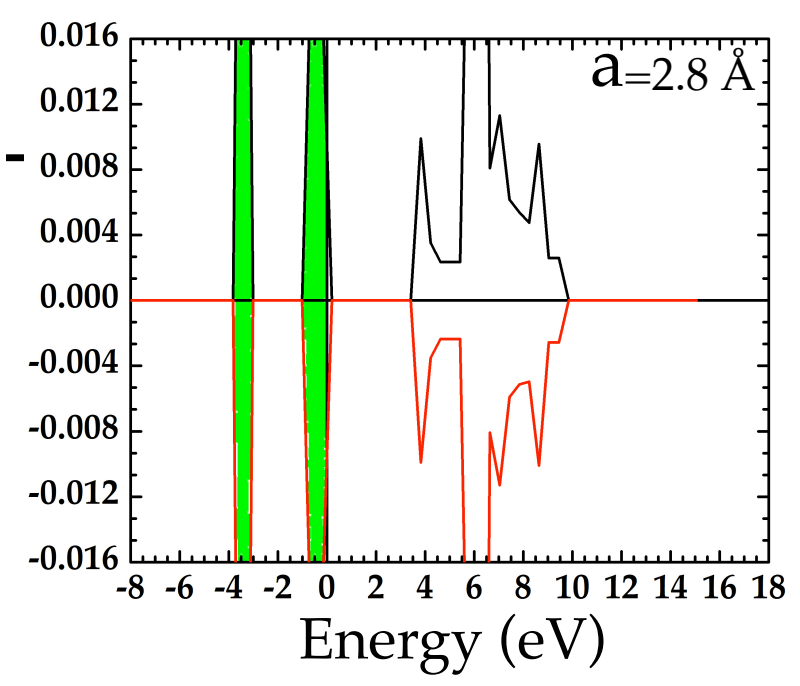

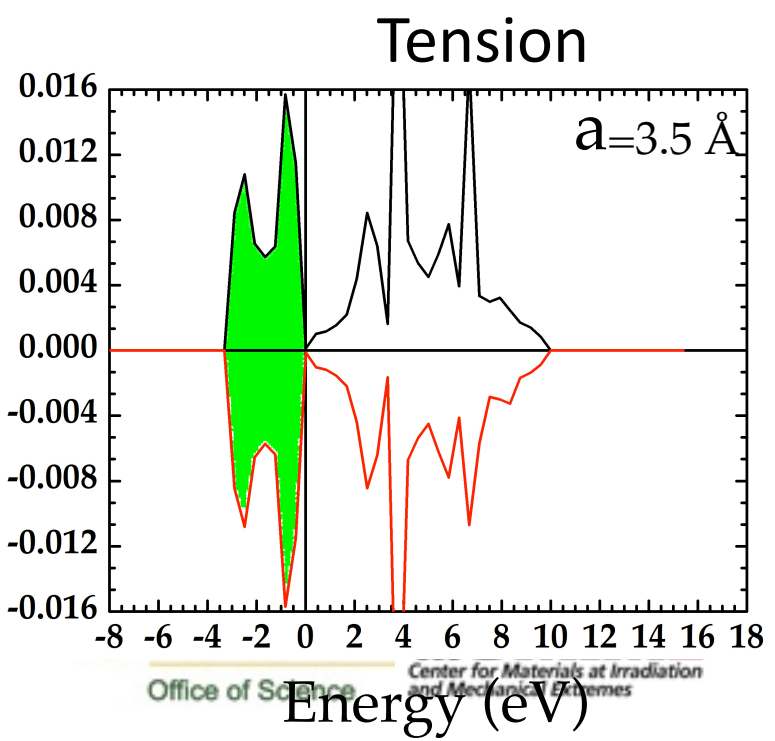




\section{$\mathrm{FCC} \mathrm{Ni}$}

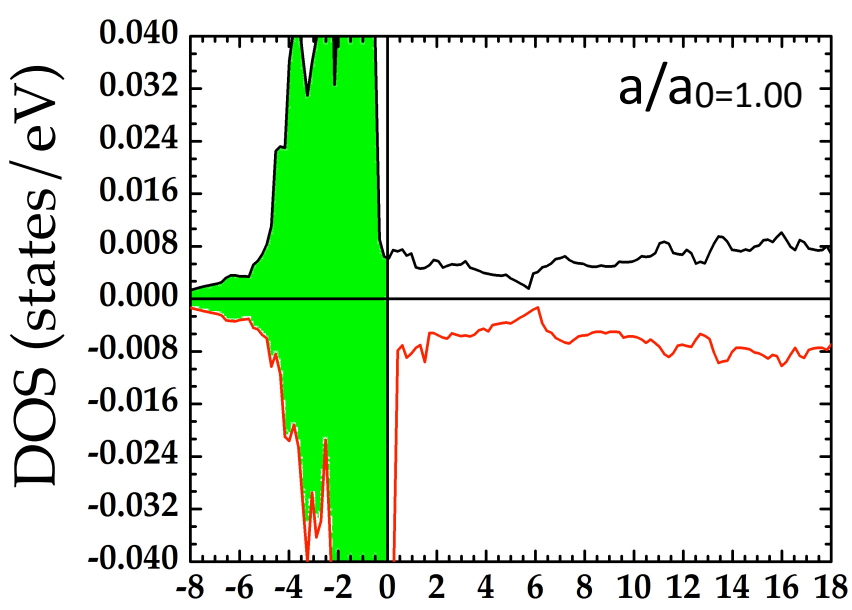

Energy (eV)

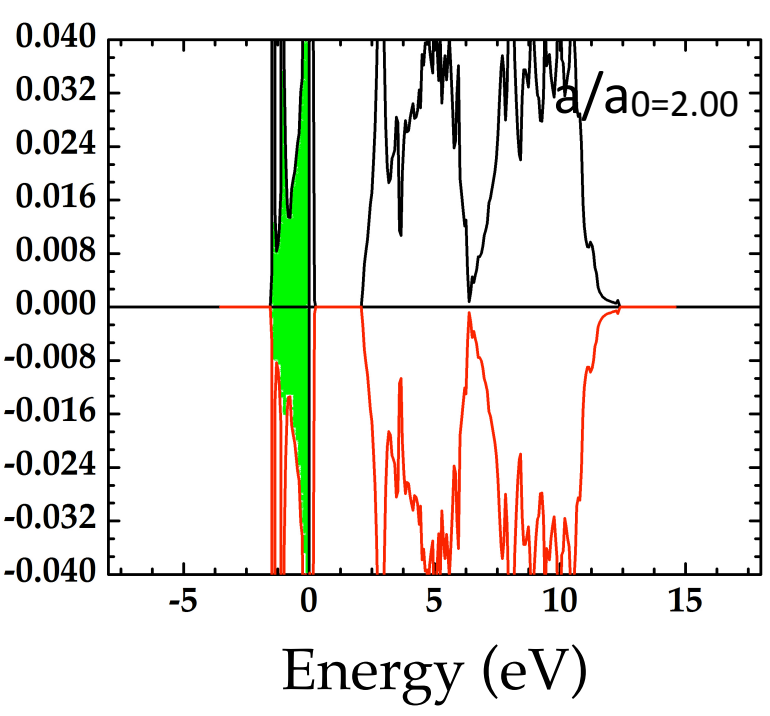

NNSA - LosAlamos 